\title{
Rosuvastatin attenuates bioprosthetic heart valve calcification
}

Sak Lee, MD, PhD, Dae-Hyun Kim, DVM, PhD, Young-Nam Youn, MD, PhD, Hyun Chel Joo, MD, Kyung-Jong Yoo, MD, PhD, and Seung Hyun Lee, MD, PhD

\section{ABSTRACT}

Objective: There are pathophysiologic similarities between calcification and atherosclerosis because both are the product of an active inflammatory process. The aim of the study was to examine the effects of statin treatment on calcification in commercially available bioprosthetic heart valves.

Methods: Twenty Sprague-Dawley rats were fed a high-fat diet to induce hypercholesterolemia during 4 weeks. They were randomly divided into 2 groups according to statin intake (control, $\mathrm{n}=10$ : high-fat diet/statin; $\mathrm{n}=10$ : high-fat diet with statin). Four commercially available tissue valve (Magna Perimount, Carpentier-Edwards, Irvine, Calif; Hancock, Medtronic, Minneapolis, Minn; Mitroflow, LivaNova, London, England; and Trifecta, St Jude Medical, St Paul, Minn) cusp samples (total 320) were implanted in rat dorsal subcutis at 4 weeks. After implantation, rosuvastatin was administered daily to the statin group. The cusps were explanted at 12 weeks, and calcium levels were determined by atomic absorption spectroscopy. Western blotting, histologic, and immunohistochemical analyses were conducted to identify the anticalcification mechanism of the statin.

Results: The mean calcium level in the control group was significantly higher than in the statin group $(P<.01)$ for all tissue valves (Magna Perimount: $2.67 \pm 0.26 \mathrm{mg} / \mathrm{g}$ vs $1.31 \pm 0.40 \mathrm{mg} / \mathrm{g}$; Hancock: $2.70 \pm 0.57 \mathrm{mg} / \mathrm{g}$ vs $1.53 \pm 0.34 \mathrm{mg} / \mathrm{g}$; Mitroflow: $2.39 \pm 0.71 \mathrm{mg} / \mathrm{g}$ vs $1.26 \pm 0.38 \mathrm{mg} / \mathrm{g}$; Trifecta: $2.54 \pm 0.42 \mathrm{mg} / \mathrm{g}$ vs $1.63 \pm 0.72 \mathrm{mg} / \mathrm{g}$ ). Inflammatory cell infiltration and interleukin- 6 and bone morphogenetic protein 2 expressions were significantly reduced in the statin group.

Conclusions: Statin treatment significantly attenuated bioprosthetic heart valve calcification associated with decreasing the levels of interleukin-6 and bone morphogenetic protein 2 . Thus, statin treatment might be helpful for the longevity of bioprosthetic heart valves. (J Thorac Cardiovasc Surg 2019;158:731-41)

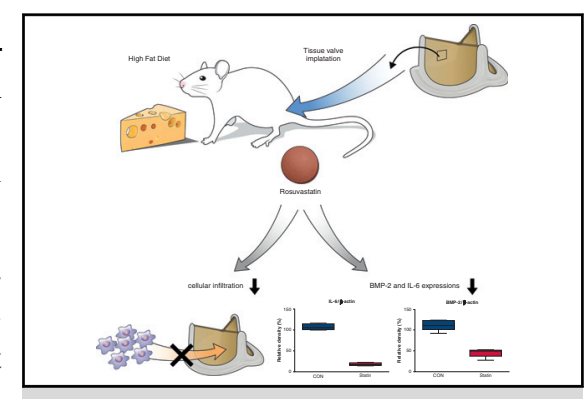

Statin attenuated bioprosthetic valve calcification.

\section{Central Message}

Statin attenuated the calcification of BHV in a rat model and should be considered for reducing BHV calcification possibly induced from inflammation.

\section{Perspective}

BHVs have the propensity to undergo structural degeneration, limiting their durability and often necessitating reoperation. Statin treatment significantly attenuated BHV calcification, which seems to be associated with decreasing the expression levels of IL-6 and BMP-2.

See Commentaries on pages 742 and 744.
Since the first implants of heterologous bioprosthetic heart valve (BHVs) in the 1960s, various improvements to tissue valve design and manufacturing have been made. BHVs

\footnotetext{
From the Division of Cardiovascular Surgery, Department of Thoracic and Cardiovascular Surgery, Severance Cardiovascular Hospital, Yonsei University Health System, Seoul, Republic of Korea.

This study was supported by a faculty research grant of Yonsei University College of Medicine (South Korea) for (6-2017-0052).

Received for publication May 9, 2018; revisions received Nov 27, 2018; accepted for publication Dec 10, 2018; available ahead of print Feb 6, 2019.

Address for reprints: Seung Hyun Lee, MD, PhD, Severance Cardiovascular Hospital, Yonsei University College of Medicine, Yonsei-ro 50-1, Seodaemun-gu, Seoul, 03722, Republic of Korea (E-mail: henry75@yuhs.ac).

0022-5223

Copyright (C) 2019 by The American Association for Thoracic Surgery. Published by Elsevier Inc. This is an open access article under the CC BY-NC-ND license (http:// creativecommons.org/licenses/by-nc-nd/4.0/).

https://doi.org/10.1016/j.jtcvs.2018.12.042
}

have central flow and a high freedom from thrombus formation and usually do not require anticoagulation treatment. However, BHVs have the propensity to undergo structural degeneration, limiting their durability and often necessitating reoperation. ${ }^{1,2} \mathrm{~A}$ study of the causes of valve failures requiring reoperation reported that sterile degeneration or primary tissue failure accounted for $74 \%$ of BHV. ${ }^{3}$ The degeneration of BHV is believed to depend on the mechanical properties of the valve and on

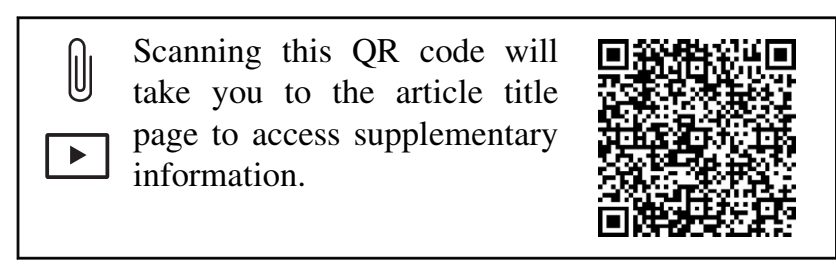




\section{Abbreviations and Acronyms \\ BHV = bioprosthetic heart valve \\ BMP-2 = bone morphogenetic protein 2 \\ IL $\quad=$ interleukin \\ LDL $=$ low-density lipoprotein \\ LLT = lipid-lowering therapy \\ Tchol $=$ total cholesterol}

immunologic and calcification processes. ${ }^{4}$ Similarities between the mechanisms and the risk factors for atherosclerosis, native valve calcification, and postoperative BHV degeneration have been proposed recently. ${ }^{3,5}$ In addition, chronic inflammatory change in the leaflet, including infiltration of macrophages and $\mathrm{T}$ lymphocytes and lipoprotein deposition in the areas of calcification, has prompted researchers to investigate whether a medical intervention can be applied to slow the progression of valvular failure. This concept has introduced a new perspective on the prevention and treatment of BHV failure. A few studies have shown that statin treatment can reduce calcific degeneration of native aortic valves. ${ }^{5-7}$ Some studies have also revealed a potential relationship between statin treatment and the durability of BHV, with statins appearing to slow the progression of $\mathrm{BHV}^{8,9}$ In our previous study, we evaluated, in a rat subdermal implantation model, the effects of a specific statin therapy, rosuvastatin (Crestor, HMG-CoA reductase inhibitor, rosuvastatin calcium, AstraZeneca Pharmaceuticals LP, Gaithersburg, Md), on postimplantation tissue changes in a commonly used bovine aortic valve bioprosthesis. ${ }^{10}$ Our results suggested that the statin could significantly attenuate the calcification of the bioprosthesis in a rat subdermal implantation model and might thereby prolong the durability of the bioprosthesis. However, only 1 type of bioprosthetic valve (a Carpentier-Edwards Perimount aortic valve, Irvine, Calif) was used in our previous experiment, so we did not obtain information about a range of commonly used tissue valves. Therefore, the aim of the present study was to evaluate the effects of statin therapy on postimplantation tissue changes in various commercially available BHVs (Video 1).

\section{MATERIALS AND METHODS}

\section{Animals, Diets, and Experimental Design}

This animal study was approved by the Institutional Animal Care and Use Committee of Yonsei University Health System (2014-0365). Twenty healthy 2-month-old Sprague-Dawley rats weighing 390.34 $\pm 12.28 \mathrm{~g}$ were used in the present study. The animals were acclimatized to a breeding room for 1 week. All 20 rats were fed a high-fat diet containing $1.25 \%$ cholesterol and $0.5 \%$ cholic acid (TD.02028, Harlan Korea Laboratories Co, Ltd, Seoul, Korea) for 12 weeks to induce hypercholesterolemia. Serum lipid levels were measured just before the high-fat diet was introduced and at 4 and 12 weeks. Samples of 4 different bioprosthetic valve cusps were surgically implanted in the dorsal subcutis at 4 weeks. The rats were then randomly allocated to the control group $(n=10$, high-fat diet only) or the statin group ( $\mathrm{n}=10$, high-fat diet with statin treatment). After implantation of the valve cusps, rosuvastatin was administered daily to the statin group. The cusps were explanted at 12 weeks, and their calcium levels were determined by atomic absorption spectroscopy. Western blotting, histologic, and immunohistochemical analyses were conducted to determine the anticalcification mechanism of the statin.

\section{Measurement of the Blood Lipid Profile and Interleukin-6 Level/Immunohistochemistry}

Nonfasting blood lipid profiles for total cholesterol (Tchol) and lowdensity lipoprotein (LDL) cholesterol were measured just before the high-fat diet was introduced and at 4 and 12 weeks for validation of hypercholesterolemia. To collect the blood, the animals were anesthetized with $10 \mathrm{mg} / \mathrm{kg}$ of xylazine and $30 \mathrm{mg} / \mathrm{kg}$ of Zoletil (Virbac, Carros, France). Then, $1.3 \mathrm{~mL}$ of blood was aseptically obtained from the subclavian vein and $0.5 \mathrm{~mL}$ of the blood sample was placed into a heparin tube, and the remaining $0.8 \mathrm{~mL}$ was placed into a serum-separating tube. Plasma samples were obtained by centrifugation at 6000 rpm for 5 minutes at room temperature. LDL cholesterol and Tchol were measured by enzymatic colorimetric assay (Modular Analytics, Roche, Germany). Serum samples were obtained by centrifugation at $14,000 \mathrm{rpm}$ for 10 minutes at $4{ }^{\circ} \mathrm{C}$ and

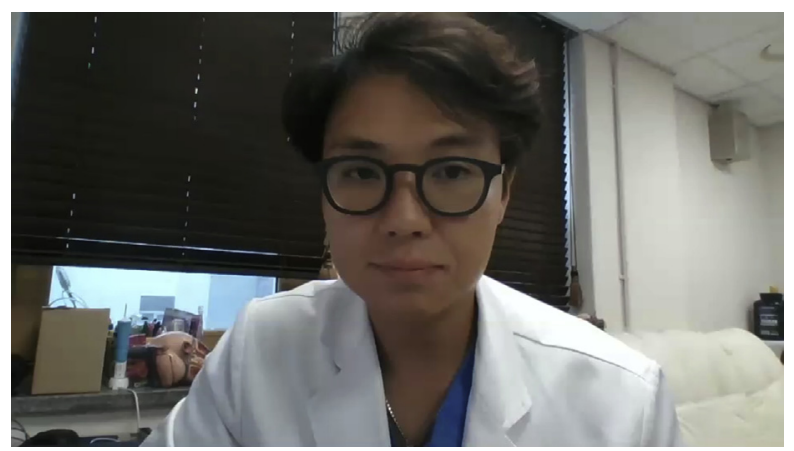

VIDEO 1. I'm Dr Seung Hyun Lee from Yonsei University (Seoul, Korea). Since the first implants of heterologous BHVs in the 1960s, various improvements to tissue valve design and manufacturing have been made. BHVs have central flow and a high freedom from thrombus formation and usually do not require anticoagulation treatment. However, BHVs have the propensity to undergo structural degeneration, limiting their durability and often necessitating reoperation. The degeneration of BHV is believed to depend on the mechanical properties of the valve and on immunologic and calcification processes. Similarities between the mechanisms and the risk factors for atherosclerosis, native valve calcification, and postoperative BHV degeneration recently have been proposed. A few studies have shown that statin treatment can reduce calcific degeneration of native aortic valves. Some studies have revealed a potential relationship between statin treatment and the durability of BHV, with statins appearing to slow the progression of BHV. In our previous pilot study, we evaluated, in a rat subdermal implantation model, the effects of a specific statin therapy, rosuvastatin (Crestor, HMG-CoA reductase inhibitor, rosuvastatin calcium, AstraZeneca Pharmaceuticals LP), on postimplantation tissue changes of bovine aortic valve bioprosthesis, and the statin significantly attenuated the calcification of the bioprosthesis in a rat subdermal implantation model. Serially, the aim of the present study was to evaluate the effects of statin therapy on postimplantation tissue changes in various commercially available BHVs. This presentation is about full process of my experiment. Video available at: https://www.jtcvs.org/article/S0022-5223(18)33481-0/ fulltext. 


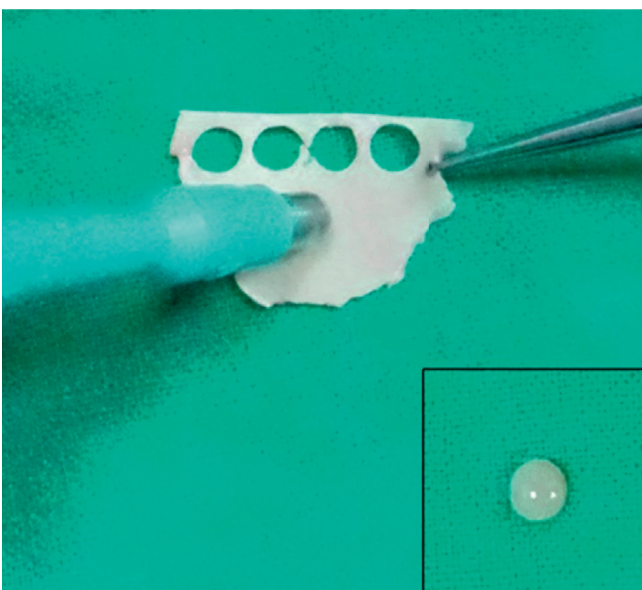

A

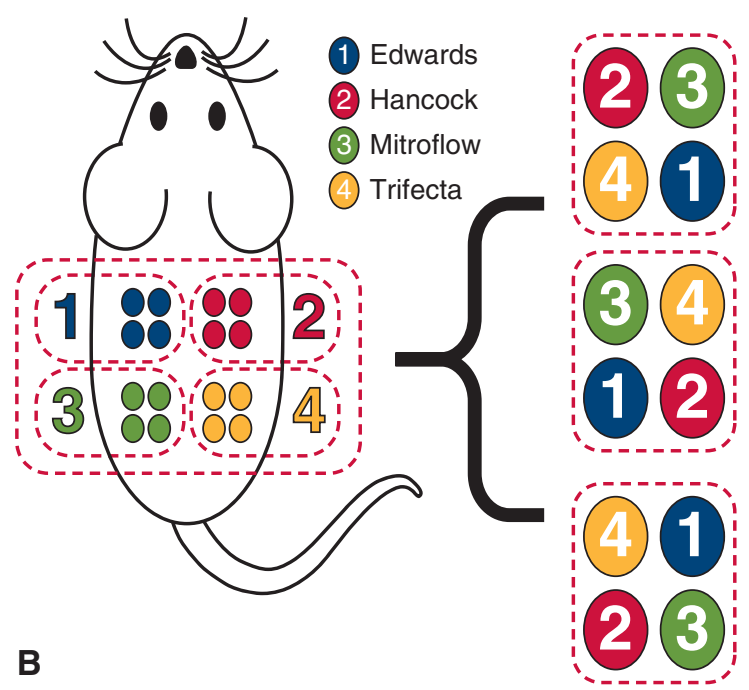

FIGURE 1. Basic schematics of the rat experimental model. A, Four different commercial BHVs were used. Three were bovine pericardial valves (Carpentier-Edwards Perimount aortic valve, Irvine, Calif; St Jude Trifecta, St Paul, Minn; and LivaNova Mitroflow, London, England), and 1 was a porcine pericardial valve (Medtronic Hancock II, Minneapolis, Minn). Each leaflet was then incised along the frame of the BHVs using a number 11 surgical blade. Then, round patches were gained using a 4-mm skin biopsy punch for implantation of rat dorsal subcutis. B, Four round patches per each commercial BHV (total 16 patches per a rat in 4 companies, each group 160 pieces, total 320 pieces) were surgically implanted into the dorsal subcutis of each rat. To avoid position effects, each BHV patch group (4 same company patches) was rotated serially in each quadrant site.

stored at $-70^{\circ} \mathrm{C}$ until further analysis. Serum interleukin (IL)-6 levels were measured at 12 weeks using enzyme-linked immunosorbent assay kits (Quantikine, Rat IL-6 Immunoassay kit, R\&D Systems, Minneapolis, Minn). Macrophages were identified by staining tissue sections with commercially available antibodies to macrophage marker MAC387 (concentration 1:100).

\section{Animal Experiment}

Four different commercial BHVs were used in this study. Three were bovine pericardial valves (Perimount, Carpentier-Edwards; Trifecta, St Jude Medical, St Paul, Minn; and Mitroflow, LivaNova, London, England), and 1 was a porcine pericardial valve (Hancock II, Medtronic Inc, Minneapolis, Minn). Each BHV was rinsed for at least 15 minutes with $500 \mathrm{~mL}$ of sterile physiologic saline solution and then transferred to another rinse basin containing sterile saline. Each leaflet was then incised along the frame of the BHV using a number 11 surgical blade. Then, round patches were gained using a 4-mm skin biopsy punch (Figure 1,A).

Four weeks after hypercholesterolemia induction, 4 round patches per BHV (total 16 patches per a rat) were surgically implanted into the dorsal subcutis of each rat. To avoid position effects, each BHV patch group (4 same company patches) was rotated serially in each quadrant site (Figure 1, B). Each animal was intraperitoneally injected with $10 \mathrm{mg} / \mathrm{kg}$ of xylazine and $30 \mathrm{mg} / \mathrm{kg}$ of Zoletil. After placing the animal in ventral recumbency, a dorsal midline skin incision was made. Then, 4 trimmed pericardial patches were implanted subcutaneously in each quadrant and fixed with a 6-0 polypropylene suture. The subcutaneous layer was sutured with a simple continuous pattern using 5-0 polyglyconate, and the skin was closed with a simple interrupted pattern using 4-0 nylon. For 3 days after surgery, $20 \mathrm{mg} / \mathrm{kg}$ of gentamicin and $1 \mathrm{mg} / \mathrm{kg}$ of meloxicam were injected subcutaneously for infection prophylaxis and analgesia, respectively.

For 8 weeks from the day after surgery, $20 \mathrm{mg} / \mathrm{kg} / \mathrm{d}$ of rosuvastatin in $2 \mathrm{~mL}$ of normal saline was given to the statin group by oral gavage using an 18-gauge feeding tube with adequate restraint. ${ }^{11}$ The rats' body weights were measured every week to calculate the drug dosage. The control group was given $2 \mathrm{~mL}$ of normal saline only.

\section{Quantitative Calcium Determination}

At 8 weeks after surgery, the animals were euthanized by $\mathrm{CO}_{2}$ inhalation and the implanted valve samples were collected. After cleanly removing the surrounding connective tissue from the implants, they were frozen for 24 hours at $-80^{\circ} \mathrm{C}$. Moisture was removed from the samples by using a freeze-dryer for 24 hours, and then the samples were weighed and then placed in $5 \mathrm{~mL}$ of aqua regia in a $100-\mathrm{mL}$ beaker. The beaker was slowly heated on a hot plate for 6 hours at $70^{\circ} \mathrm{C}$ to $80^{\circ} \mathrm{C}$ to avoid tissue disruption from rapid thawing and rewarming, followed by 5 hours at $140^{\circ} \mathrm{C}$ to $150^{\circ} \mathrm{C}$; $5 \mathrm{~mL}$ of $\mathrm{H}_{2} \mathrm{O}_{2}$ was then added. The calcium content of the samples was analyzed using a Perkin Elmer Optima 8300 ICP-OES spectrometer (Norwalk, Conn).

\section{Histopathologic and Immunohistochemical Evaluation}

Extracted BHV tissue samples were fixed in $10 \%$ neutralized buffered formalin (BBC Biochemical, Mount Vernon, Wash) and processed using standard methods and embedded in paraffin. Sections $4-\mu \mathrm{m}$ thick were stained with hematoxylin (Sigma-Aldrich, St Louis, Mo) and eosin (BBC Biochemical). For immunohistochemistry, the sections were deparaffinized using standard protocols, and antigen retrieval was performed with

TABLE 1. Intergroup comparison of the lipid profile results

\begin{tabular}{lccc}
\hline & Statin group & Control group & $P$ valve \\
\hline Tchol (pre-) (mg/dL) & $70.80 \pm 7.50$ & $72.40 \pm 11.04$ & NS \\
LDL (pre-) (mg/dL) & $14.70 \pm 3.47$ & $15.10 \pm 3.96$ & NS \\
Tchol (4 wk) (mg/dL) & $280.10 \pm 97.17$ & $267.80 \pm 61.15$ & NS \\
LDL (4 wk) (mg/dL) & $201.50 \pm 80.86$ & $190.40 \pm 57.41$ & NS \\
Tchol (12 wk) (mg/dL) & $181.50 \pm 57.12$ & $257.30 \pm 30.10$ & $<.01$ \\
LDL (12 wk) (mg/dL) & $130.10 \pm 49.35$ & $227.50 \pm 57.00$ & $<.01$ \\
\hline
\end{tabular}

Tchol, Total cholesterol; NS, not significant; $L D L$, low-density lipoprotein. 

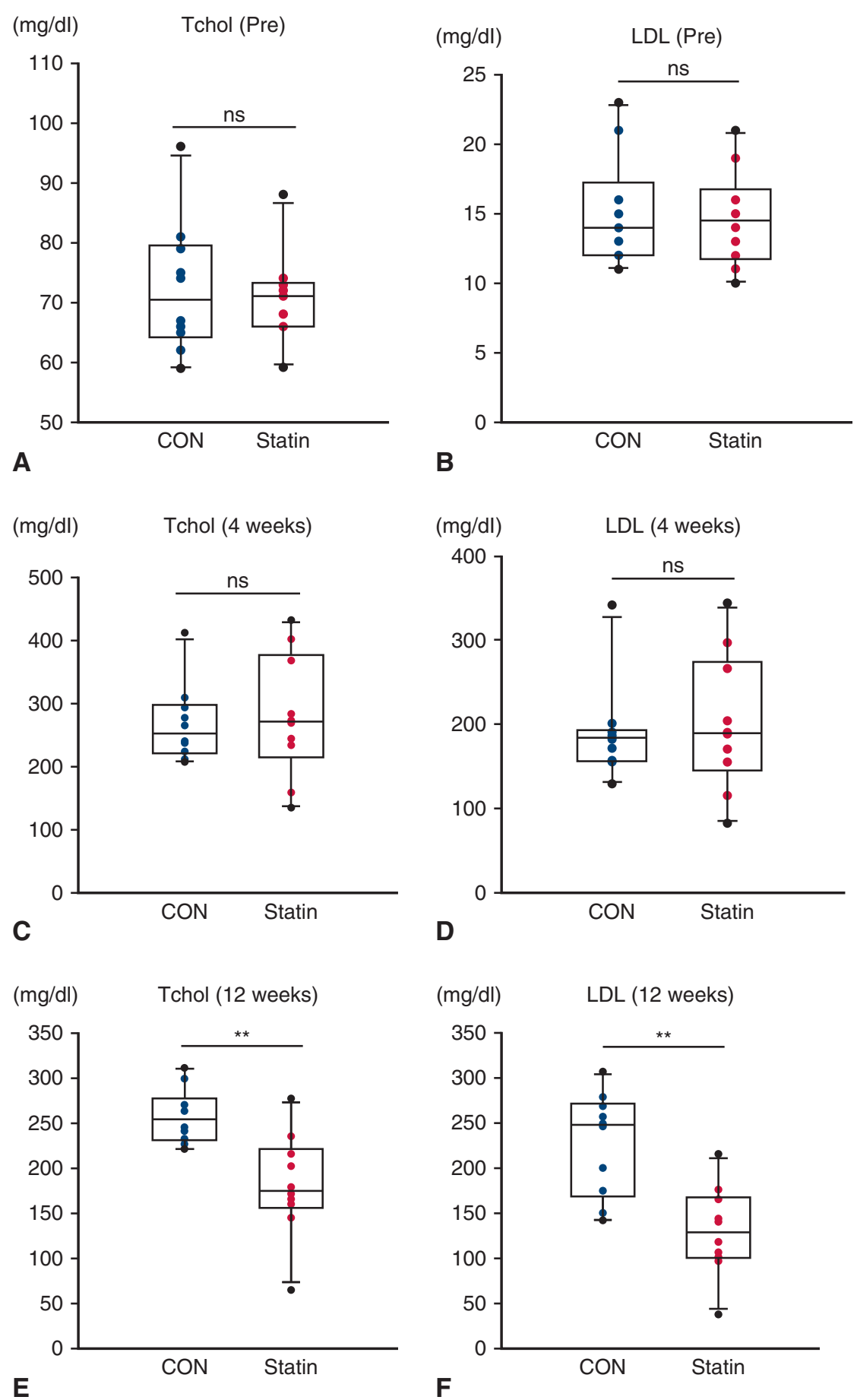

FIGURE 2. Lipid profile difference between groups and change through experiment. All 20 rats were fed a high-fat diet for 12 weeks to induce hypercholesterolemia. Serum lipid levels were measured just before the high-fat diet was introduced and at 4 and 12 weeks. Samples of 4 different bioprosthetic valve cusps were surgically implanted in the dorsal subcutis at 4 weeks. For checking adequate hyperlipidemia in all rats, serum lipid levels (ex), Tchol level, and LDL were checked at 4 weeks. After implantation, the statin group was given $20 \mathrm{mg} / \mathrm{kg} / \mathrm{d}$ rosuvastatin in $2 \mathrm{~mL}$ of normal saline: control group ( $\mathrm{n}=10$, highfat diet only) or statin group ( $\mathrm{n}=10$, high-fat diet with statin treatment). Statin group showed a significantly low lipid profile after 12 weeks. A, Preimplantation Tchol level was similar in groups (control, $\mathrm{n}=10$ vs statin, $\mathrm{n}=10$ ). Preimplantation Tchol level between statin and control groups: $70.80 \pm 7.50$ versus $72.40 \pm 11.04$ (control box: 65.25-78, median: 70.5, 59 96/statin box: 66.5-72.75, median: 71, 59 88). B, Preimplantation LDL level was similar in groups (control, $\mathrm{n}=10$ vs statin, $\mathrm{n}=10$ ). Preimplantation LDL level between statin and control groups: $14.70 \pm 3.47$ versus $15.10 \pm 3.96$ (control box: 12.5-15.75, median: 14, 11 23/statin box: 12.25-16, median: 14.5, 10 21). C, Both groups showed appropriate hyperlipidemia status (control, $\mathrm{n}=10$ vs statin, $\mathrm{n}=10$ ). Postimplantation (4 weeks) Tchol level (just before initiation of statin treatment) between statin and control groups: 
Tris-EDTA ( $\mathrm{pH}$ 9.0) buffer. Then, sections were incubated for 10 minutes in 3\% hydrogen peroxide (Duksan Hydrogen Peroxide 3059, Kyungkido, Korea) to inactivate endogenous peroxidase and blocked by incubating for 1 hour in $5 \%$ bovine serum albumin. Subsequently, the sections were labeled with the following primary mouse monoclonal antibodies (Abcam, Cambridge, United Kingdom: anti-IL-6) (ab6672), antimacrophage (MAC387, ab22506), and anti-bone morphogenetic protein 2 (BMP-2) (ab6285). The antibody-labeled sections were then incubated with Dako EnVision+ System-HRP-labeled polymer antimouse kit solution (Dako4001, Dako, Glostrup, Denmark). DAP (K-3468, Dako) staining was performed for 3 minutes at room temperature for tissue visualization. Sections were then counterstained with hematoxylin.

Eight pictures of each sample were obtained at $\times 400$ magnification and used for cell counts. Counts of infiltrated cells or immunostaining positive cells within the implants were performed blindly by an objective pathologist.

\section{Western Blot Analysis}

To investigate the expression of IL-6 and BMP-2, samples were stored at $-70^{\circ} \mathrm{C}$. Protein was extracted with $500 \mu \mathrm{L}$ of PRO-PREP protein extraction solution (Intron Biotechnology, Kyungki-Do, Korea), followed by tissue homogenization using sharp scissors. After 30 minutes on ice, the samples were centrifuged at $14,000 \mathrm{rpm}$ for 10 minutes at $4^{\circ} \mathrm{C}$. The supernatant containing the protein lysates was then collected.

The protein content was quantified using a protein-assay kit (Bio-Rad, Hercules, Calif). For immunoblotting, $50 \mu \mathrm{g}$ of total protein extract was mixed with Laemmli sample buffer and transferred to a Mini Protean TGX precast gradient gel (Bio-Rad) with DOKDO-MARK (Intron Biotechnology) as the standard prestained protein marker. Gel electrophoresis was performed with a Mini Protean system (Bio-Rad) applying $100 \mathrm{~V}$ for 130 minutes. After electrophoresis, proteins were blotted to a methanol preactivated polyvinylidene difluoride membrane applying $230 \mathrm{mAh}$ for 90 minutes using a Mini Trans-Blot Cell (Bio-Rad). The blotted membranes were then blocked and incubated with anti-BMP-2 antibody (ab6285, 1:1000 dilution, Abcam) and anti-IL-6 antibody (ab6672, $1: 1000$ dilution, Abcam) in PBS-T with $5 \%$ skimmed milk. Anti- $\beta$-actin antibody was used as a loading control (ADI-905-733, 1:2000 dilution, Enzo Life Sciences, New York, NY). After incubation with the primary antibodies, the membranes were washed with PBS-T buffer 3 times and then incubated with secondary goat anti-mouse immunoglobulin-G horseradish peroxidase (ab6789, 1:2000 dilution, Abcam) for the anti-BMP-2 antibody and anti-Il6 antibody and goat anti-rabbit immunoglobulin-G horseradish peroxidase (ADI-SAB-300-J, 1:2000 dilution, Enzo Life Sciences) for the anti- $\beta$-actin antibody. Proteins were detected using an enhanced chemiluminescence detection reagent for immunoblot analysis (Daeill Lab Service, Seoul, Korea). Subsequently, quantitative analysis was performed with the pixel density analysis method using Image $\mathbf{J}$ software (National Institutes of Health, Bethesda, Md). Relative density was defined as (specific band density $/ \beta$-actin band density) $\times 100$.

\section{Statistical Analysis}

All data were expressed as mean \pm standard deviation. Statistical analyses were performed using GraphPad Prism 5.0 software (GraphPad
Software, Inc, San Diego, Calif). Normal data distribution was determined using the Shapiro-Wilk test. The Student $t$ test was used to compare mean blood lipids, Il-6, and BMP-2 expression levels, tissue calcium level, and the relative density of Western blotting level between groups (statin vs control). For comparison of 4 different valve types, analysis of variance test was used. The Kruskal-Wallis test and Mann-Whitney test were applied to test overall/pairwise group mean differences in calcium levels.

\section{RESULTS \\ Effect of Rosuvastatin on Blood Lipid Profiles and Body Weight}

Rosuvastatin significantly reduced the blood Tchol levels in hypercholesterolemic animals. The Tchol levels of the 2 groups before administration of the high-fat diet were not significantly different (Table 1, Figure 2, A). At 4 weeks, the Tchol levels were significantly increased compared with the initial levels, and there was no significant difference between the groups (Table 1, Figure 2, C). However, at 12 weeks the Tchol level of the statin group was significantly lower than that of the control group $(P<.001$, Table 1, Figure 2,E). The effect of rosuvastatin on blood LDL cholesterol level was similar. The LDL cholesterol levels at the beginning of the experiment and at 4 weeks were not significantly different between the groups (Table 1, Figure 2, $B$ and $D$ ), but at 12 weeks the statin group had significantly lower LDL cholesterol than the control group $(P<.001$, Table 1, Figure $2, F)$. Rosuvastatin did not affect the animals' body weight change. All of the animals gained weight every week, and there were no significant differences between the groups.

\section{Effect of Rosuvastatin on Calcium Levels in the Implants}

The calcium levels in the tissue valve samples were measured 12 weeks after implantation. The calcium level of the control group was significantly higher than that of the statin group $(P<.01)$ regardless of BHV type (Table 2, Figure 3, $A-D$ ). There was no significant difference in the calcium levels of the different BHV types $(P=.62$, Figure E1).

\section{Effect of Rosuvastatin on Cellular Infiltration of the Bovine Pericardial Implants}

Rosuvastatin significantly reduced cellular infiltration of the pericardial implants in the hypercholesterolemic animals. Numerous cellular infiltrates were observed around

$280.10 \pm 97.17$ versus $267.80 \pm 61.15$ (control box: 227.5-289.75, median: 252.5, 208 412/statin box: 236.5-347, median: 271.5, 135 432). D, Both groups showed appropriate hyperlipidemia status (control, $\mathrm{n}=10$ vs statin, $\mathrm{n}=10$ ). Postimplantation ( 4 weeks) LDL level (just before initiation of statin treatment) between statin and control groups: $201.50 \pm 80.86$ versus $190.40 \pm 57.41$ (control box: 160.75-190.25, median: 184.5, 129 342/statin box: 159251.25, median: 190, 82 344). E, Statin group showed effectively decreased Tchol level (control, $\mathrm{n}=10 \mathrm{vs}$ statin, $\mathrm{n}=10$ ). Postimplantation (12 weeks) Tchol level (after statin treatment) between statin and control groups: $181.50 \pm 57.12$ versus $257.30 \pm 30.10, P<.01$ (control box: $234.25-268.5$, median: 254, 221 311/statin box: 160.75-212.5, median: 175, 65 277). F, Statin group showed effectively decreased LDL level (control, $\mathrm{n}=10$ vs statin, $\mathrm{n}=10$ ). Postimplantation (12 weeks) LDL level (after statin treatment) between statin and control group: $130.10 \pm 49.35$ versus $227.50 \pm 57.00, P<.01$ (control box: 181.25-266, median: 248, 142 307/statin- box: 103-159.75, median: 129, 38 215). LDL, Low-density lipoprotein; Tchol, total cholesterol. 
TABLE 2. Tissue calcium level in the different prostheses

\begin{tabular}{lccc}
\hline & \multicolumn{3}{c}{ Calcium level } \\
\cline { 2 - 4 } & Statin group & Control group & $\boldsymbol{P}$ value \\
\hline $\begin{array}{c}\text { Edwards (mg/g) (Irvine, } \\
\quad \text { Calif) }\end{array}$ & $1.31 \pm 0.40$ & $2.67 \pm 0.26$ & $<.01$ \\
Trifecta (mg/g) (St Jude & $1.63 \pm 0.72$ & $2.54 \pm 0.42$ & $<.01$ \\
$\quad$ Medical, St Paul, Minn) & & & \\
Mitroflow (mg/g) (LivaNova, & $1.26 \pm 0.38$ & $2.39 \pm 0.71$ & $<.01$ \\
$\quad$ London, England) & & & \\
$\begin{array}{c}\text { Hancock (mg/g) (Medtronic } \\
\text { Inc, Minneapolis, Minn) }\end{array}$ & $1.53 \pm 0.34$ & $2.70 \pm 0.57$ & $<.01$ \\
\hline
\end{tabular}

the implants, and some of them were also present within the fibers of the implants. More cellular infiltration was observed in the control group than in the statin group (control vs statin: $121.30 \pm 23.59$ vs $85.30 \pm 11.22, P<.01$, Figure 4).

\section{Effect of Rosuvastatin on the Expression of the Proteins Involved in Calcification Metabolism and on Cell Populations}

The immune-staining results showed that the positive cell count ratios (positive cell/total cell, \%) for calcification
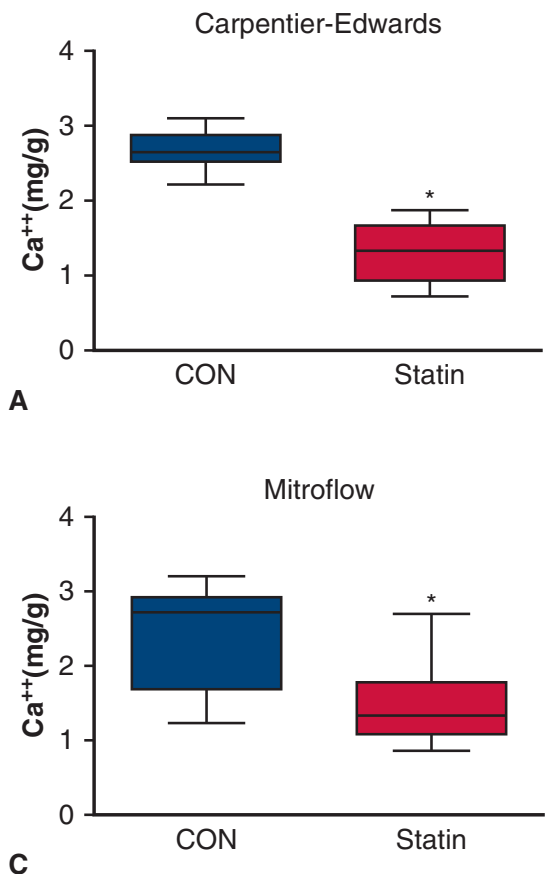

induction BMP-2 and the inflammatory marker IL-6 were significantly higher in the control group (control vs statin: $22.94 \% \pm 4.54 \%$ vs $9.97 \% \pm 1.15 \%, P<.01$; $21.82 \% \pm 8.19 \%$ vs $5.06 \% \pm 5.96 \%, P=.02$; and $34.63 \% \pm 6.25 \%$ vs $10.37 \% \pm 3.23 \%, P<.01$, respectively) (Figure 5) (MAC 387: the monoclonal antibody, which show a similar reaction pattern as antisera to a complex formed by the calcium-binding proteins. Macrophages were identified by staining tissue sections with commercially available antibodies). The relative band density of each group is shown in Figure 6, A. Western blotting revealed that BMP-2 and IL-6 expressions were significantly increased in the control group. However, rosuvastatin significantly suppressed BMP-2 (109.66\% $\pm 8.98 \%$ vs $16.52 \% \pm 3.47 \%, P<.01)$ and IL-6 $(113.08 \% \pm$ $17.78 \%$ vs $42.34 \% \pm 12.98 \%, P<.01$ ) (Figure $6, B$ and $C$ ).

\section{DISCUSSION}

Our previous pilot study investigated the effect of rosuvastatin on the calcification of bovine pericardial aortic tissue valves in a rat subdermal implantation model. ${ }^{10}$ The principal finding of that study was that high cholesterol levels may be a significant risk factor for bovine pericardial valve calcification. Statin treatment was able to
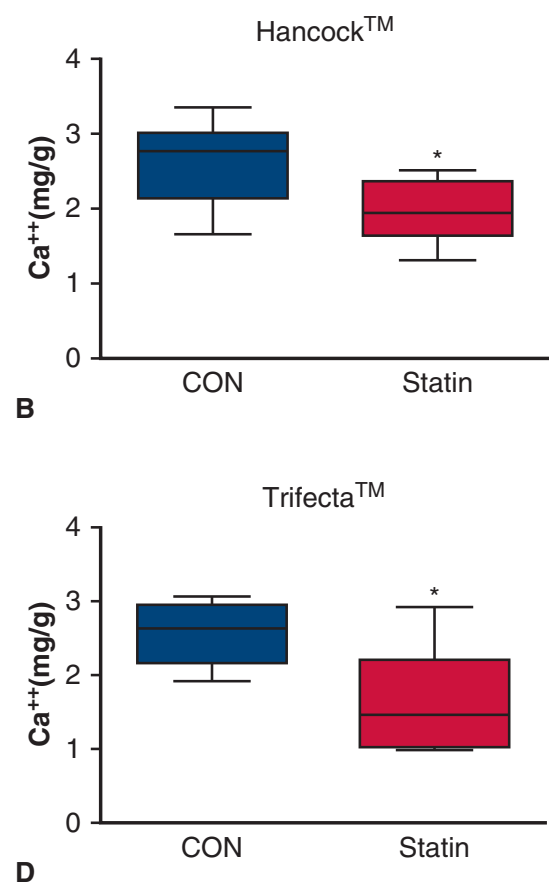

FIGURE 3. The calcium levels in the extracted BHV samples were measured 12 weeks after implantation. The calcium level of the control group was significantly higher than that of the statin group (control, $\mathrm{n}=160$ vs statin, $\mathrm{n}=160, P<.01$ ) regardless of BHV type. A, Carpentier-Edwards Perimount aortic valve: $1.31 \pm 0.40$ versus $2.67 \pm 0.26, P<.01$ (statin, $\mathrm{n}=40$ vs control, $\mathrm{n}=40$ ) (control box: $2.54-2.81$, median: 2.64, 2.21 3.1/statin box: 1.00-1.60, median: $1.33,0.72 \sim 1.87$ ). B, Hancock aortic valve: $1.53 \pm 0.34$ versus $2.70 \pm 0.57, P<.01$ (statin, $\mathrm{n}=40$ vs control, $\mathrm{n}=40$ ) (control box: $2.20-2.9$, median: 2.76, 1.66 3.35/statin box: 1.69-2.23, median: 1.94, 1.31 2.51). C, Mitroflow aortic valve: $1.26 \pm 0.38$ versus $2.39 \pm 0.71, P<.01$ (statin, $\mathrm{n}=40$ vs control, $\mathrm{n}=40$ ) (control box: 1.72-2.86, median: 2.72, 1.23 3.2/statin box: 1.13-1.69, median: 1.33, 0.86 2.69). D, Trifecta aortic valve: $1.63 \pm 0.72$ versus $2.54 \pm 0.42, P<.01$ (statin, $\mathrm{n}=40$ vs control, $\mathrm{n}=40$ ) (control box: $2.23-2.89$, median: $2.63,1.91 \sim 3.07 /$ statin box: 1.04-1.92, median: $1.46,0.98 \sim 2.92)$. 

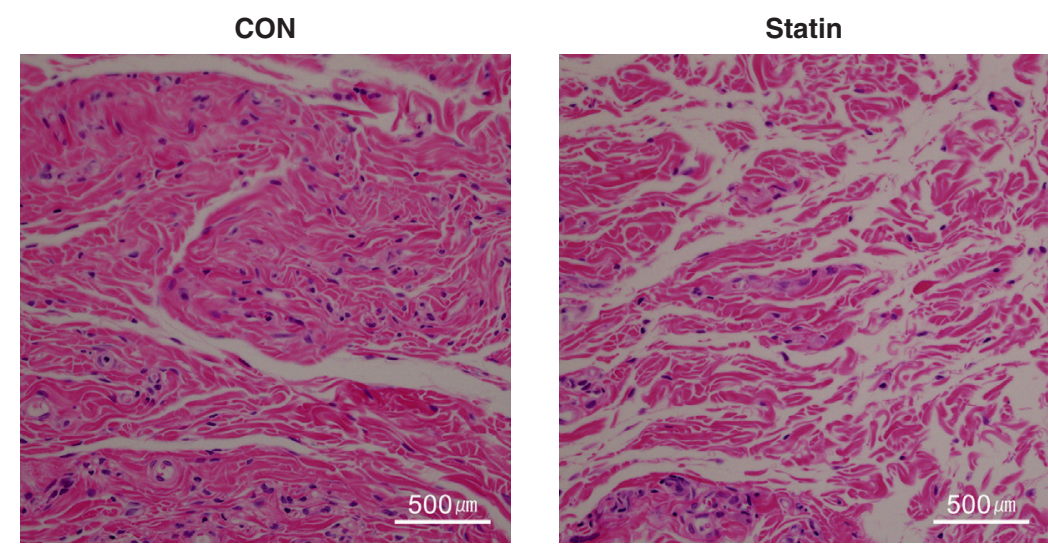

H\&E

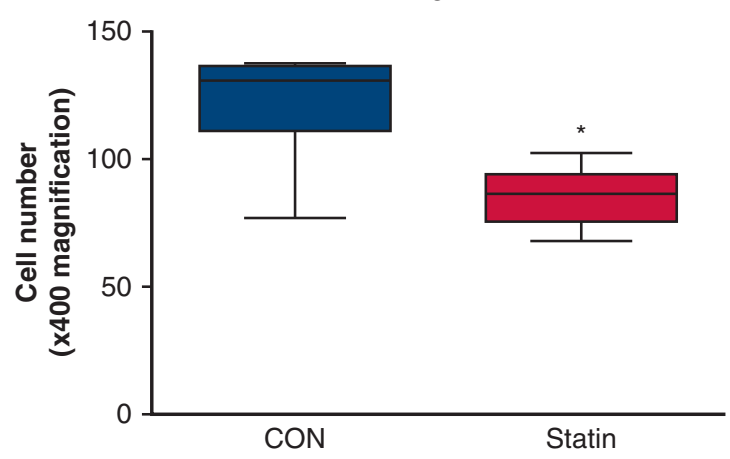

FIGURE 4. Rosuvastatin significantly reduced cellular infiltration of the BHV in the hypercholesterolemic rats. Numerous inflammatory cell infiltrates were observed around the implants, and some of them were also present within the fibers of the implants in control group (upper left); however, the statin group showed near normal cellular concentration and distribution (upper right) More inflammatory cellular infiltration was observed in the control than in the statin group (control, $\mathrm{n}=160$ vs statin, $\mathrm{n}=160: 121.30 \pm 23.59$ vs $85.30 \pm 11.22, P<.01$ ) (lower figure). H\&E, Hematoxylin-eosin.

significantly attenuate calcification of bovine pericardial valves in a subcutaneous rat model and had the dual effect of anticalcification and anti-inflammation regardless of hypercholesterolemia. However, our previous experiment was performed using just one type of bioprosthesis (CarpentierEdwards Perimount aortic valve). Therefore, we wanted to evaluate the effect of statin treatment on other popular types of bioprostheses, including one made with a different source material (the Medtronic Hancock II valve made of porcine pericardial tissue), and to re-verify statin's anticalcification and anti-inflammation effects on bovine pericardial bioprostheses. To simplify the study groups, we used only the hypercholesterolemia rat model for proving the statin effect on bioprosthesis in a recent experiment. In our previous experiment, to clearly show the effect of statin treatment, we classified animals into 4 groups according to hypercholesterolemia and statin intake to observe the effect of statin treatment on both nonhyperlipidemia and hyperlipidemia. Our results showed a strong effect of statin treatment for preventing hypercholesterolemia and antiinflammatory reactions from inhibition of cellular infiltrates and IL-6 activation (Figure 7). Also, normal calcium deposition was found in both statin treatment groups regardless of cholesterol level.

Farivar and $\mathrm{Cohn}^{5}$ reported that increasing serum cholesterol levels are associated with increased BHV calcification. They found that patients who had their valves explanted had higher serum cholesterol levels than an age, survival duration, and valve-position-matched group with nonexplanted valves ( $189 \mathrm{vs} 163 \mathrm{mg} / \mathrm{dL}, P<.001)$, and the odds ratio for valve explantation was 3.9-fold higher in patients whose serum cholesterol levels were greater than $200 \mathrm{mg} / \mathrm{dL}$. Palta and colleagues ${ }^{8}$ also reported that patients with a serum cholesterol level greater than $200 \mathrm{mg} / \mathrm{dL}$ had a native aortic valve area reduction rate approximately twice that of those with a lower cholesterol level $(P=.04)$. However, based on a review of published studies, Gilmanov and colleagues ${ }^{12}$ found that although the data are conflicting, statin therapy does not prevent structural valve deterioration of $\mathrm{BHV}$ in the aortic position. Likewise, Bellamy and colleagues ${ }^{13}$ found no correlation between the rate of progression of aortic stenosis and cholesterol levels in 157 patients, although statin treatment was associated with slower progression: Aortic valve area decreased by $3 \mathrm{~mm} \pm 10 \%$ 

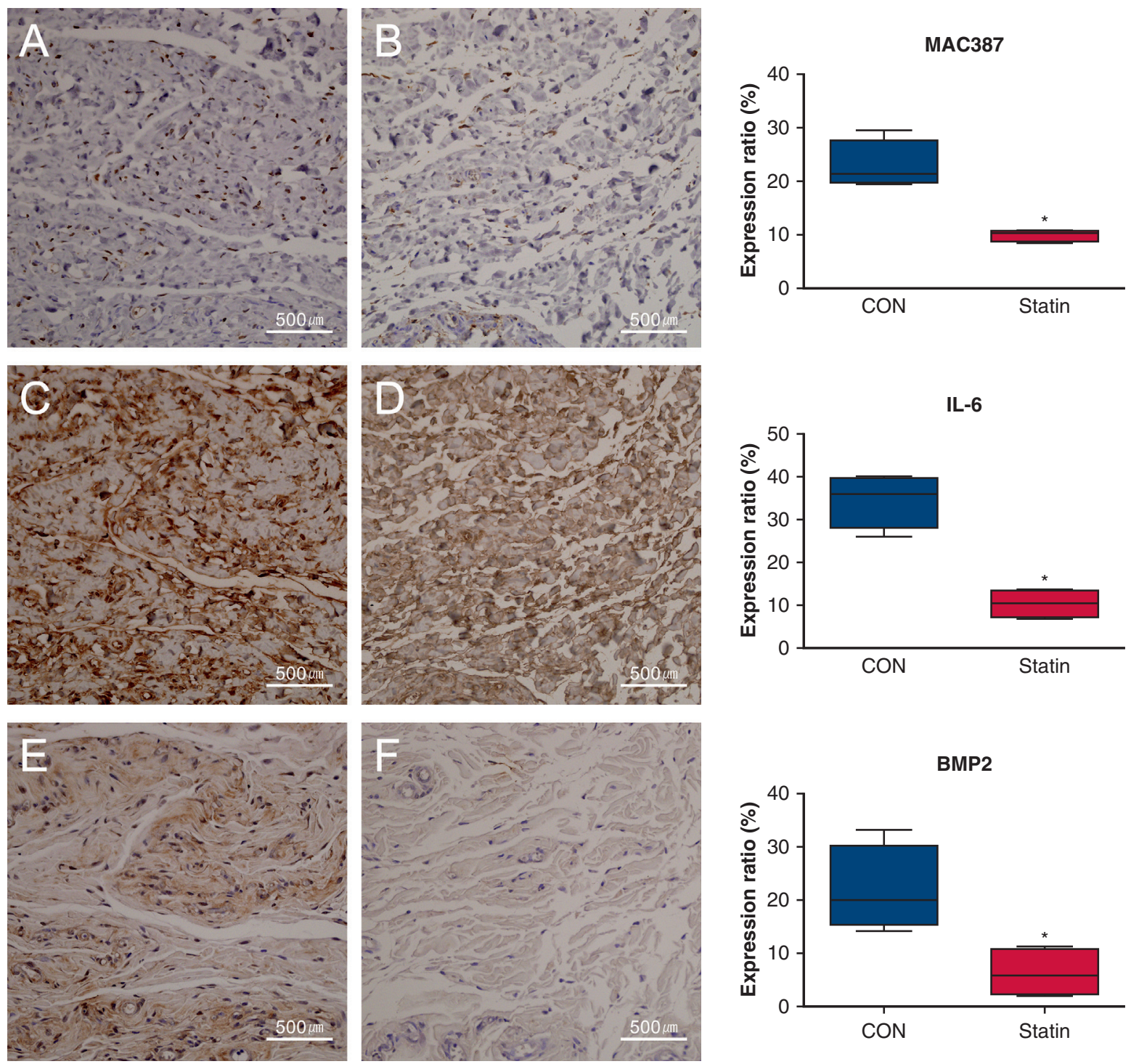

FIGURE 5. The immune-staining results in BHV showed that the positive cell count ratios (positive cell/total cell, \%) for calcification induction (MAC 387, BMP-2) and the inflammatory marker IL-6 were significantly higher in the control group (control, $\mathrm{n}=160 \mathrm{vs}$ statin, $\mathrm{n}=160$ ). This result showed statin can reduce both calcification and inflammation. A and B, MAC 387, 22.94\% $\pm 4.54 \%$ (control) versus $9.97 \% \pm 1.15 \%$ (statin), $P<.01$ (control box: 20.5 23.82, median: 21.42, 19.3 29.5/statin box: 9.58-10.71, median: 10.33, 8.3 10.8). C and D, Il-6, 34.63\% $\pm 6.25 \%$ (control) versus $10.37 \% \pm 3.23 \%$ (statin), $P<.01$ (control box: 32.2-38.3, median: 36.03, 26.0 40.4/statin box: 8.08-12.7, median: 10.5, 6.8 13.6). E and F, BMP-2, 21.82\% $\pm 8.19 \%$ (control) versus 5.06\% $\pm 5.96 \%$ (statin), $P=.02$ (control box: 17.8-23.8, median: 19.95, 14 33.3/statin box: 0-9.4, median: 4.34, 0 11.5). MAC, Antimacrophage; $I L$, interleukin; $B M P$-2, bone morphogenetic protein 2.

per year in patients receiving statins compared with $7 \mathrm{~mm} \pm 13 \%$ per year in untreated patients. Kulik and colleagues ${ }^{14}$ analyzed 1193 patients who underwent aortic valve replacement with a contemporary bioprosthesis, 150 of whom received lipid-lowering therapy (LLT) early after surgery and were compared with patients who did not receive LTT. They found no association between early postoperative LLT and a slowing of bioprosthesis structural valve degeneration, and concluded that with the excellent durability of $\mathrm{BHV}$ in the current era, a prospective randomized trial of statin therapy to prevent bioprosthetic structural valve degeneration does not appear to be justified, let alone feasible. ${ }^{14}$ Unfortunately, until now, no clinical trials have been run long enough to determine if statins have a significant benefit in preventing structural valve deterioration, and all of the retrospective studies are confounded by hypercholesterol (or coronary artery disease) indications for statin initiation. A recent animal experimental study ${ }^{15}$ using bovine pericardial bioprostheses in a rat subdermal implantation model showed that atorvastatin treatment 

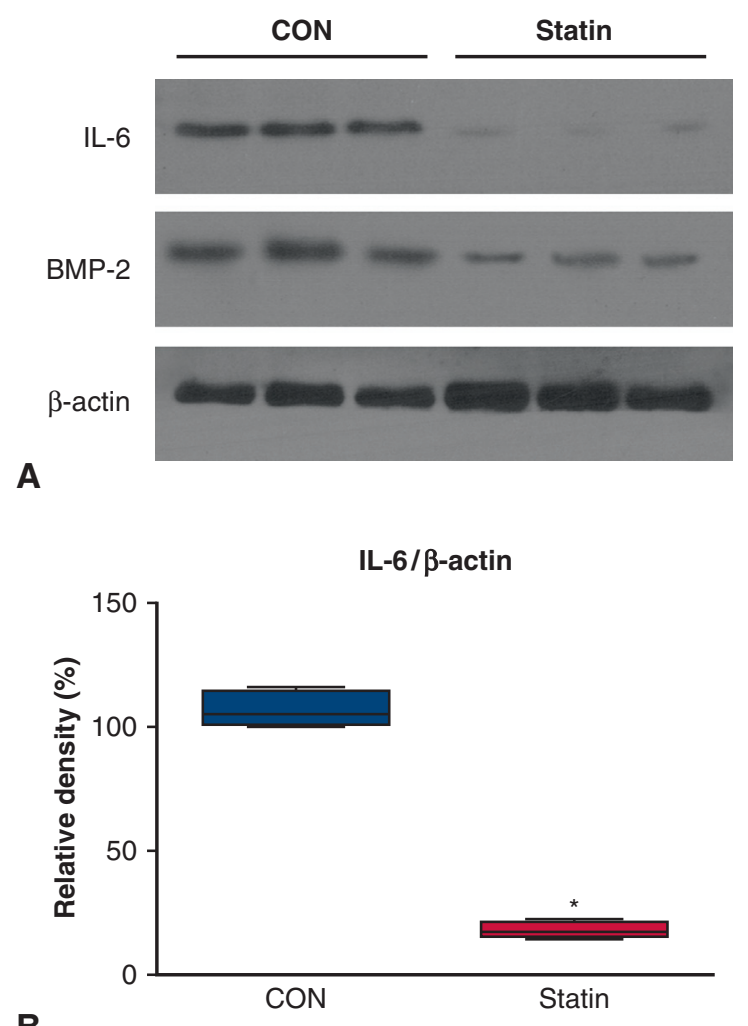

B

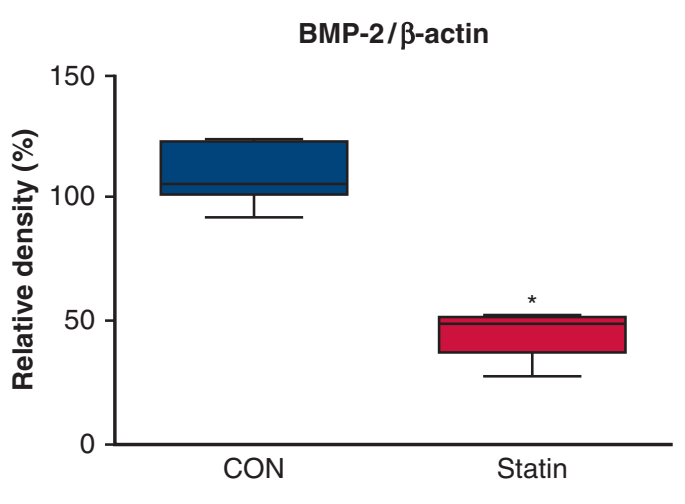

C

FIGURE 6. Western blotting in BHV results for inflammatory markers. A, The relative band density of each group. Western blotting revealed that BMP-2 and IL-6 expressions were significantly increased in the control group. However, rosuvastatin significantly suppressed BMP-2 (control, $\mathrm{n}=160$ vs statin, $\mathrm{n}=160$ ). A, Band density of each group for inflammatory markers and BMP-2. B, IL-6, 113.08\% $\pm 17.78 \%$ (control) versus $42.34 \% \pm 12.98 \%$ (statin), $P<.01$. C, BMP-2 109.66\% $\pm 8.98 \%$ (control) versus $16.52 \% \pm 3.47 \%$ (statin), $P<.01$. IL, Interleukin; $B M P-2$, bone morphogenetic protein 2 .

attenuated calcification of the bioprosthesis and reduced the global extent of inflammatory infiltrates. Lorusso and colleagues ${ }^{15}$ suggested that atorvastatin significantly attenuates the postimplantation structural degeneration of bovine valve tissue in a subcutaneous animal model.
However, the correlation between lipid deposition and bioprosthesis calcification was not clear, because the hypercholesterolemia condition was not investigated. Because hypercholesterolemia is the optimal condition in which to observe a correlation between lipids and BHV, we adopted the concept of the previous experiment and made a high lipid-profile rat model. In our previous study, we suggested that hypercholesterolemia is clearly associated with BHV calcification. Moreover, our results indicated that statin treatment can prevent postimplant calcification and inflammatory infiltration in an animal model. ${ }^{10}$ It has been reported that cholesterol deposition may play an important role in long-term structural valve degeneration even in individuals with normal lipid profiles. The deposition of LDL-C particles on the external surface of the valve leaflets initiates a chronic inflammatory reaction, which, over a long period of time, leads to calcification, scarring, and leaflet dysfunction. ${ }^{16}$

In real-world situations, there are various types of BHV for heart valve surgery, and we could not conclude from our previous experiment that statin treatment has the same effect on bovine and porcine pericardial tissue valves. Therefore, we included different types of BHV in this experiment and re-verified the effect on bovine pericardial valve tissue (including the Carpentier-Edwards Perimount aortic valve). Our results revealed that all of the BHVs showed a similar calcification tendency regardless of statin intake (Figure 3). The correlation of increased cholesterol levels with BHV calcification leads to speculation about the mechanism involved. ${ }^{17}$ Additionally, oxidized LDL cholesterol, which interacts with $\mathrm{T}$ lymphocytes and calcium, has been demonstrated in degenerative aortic valve stenosis. ${ }^{18}$ The hypothesis of pathophysiologic similarity among atherosclerosis, bioprosthetic degeneration, and native valve degeneration has recently been suggested, indicating new directions of research, particularly into treatment and prevention options. ${ }^{8,9,19}$ However, the role of hypercholesterolemia in BHV degeneration remains unclear. Lorusso and colleagues ${ }^{14}$ reported a clear reduction of calcium deposition in the pericardial tissue explanted from animals on statin therapy, in terms of both microcalcification and global calcium content. Our previous results revealed strong effects of statin treatment in preventing hypercholesterolemia and promoting an anti-inflammatory reaction, as proven by inhibition of cellular infiltrates and IL-6 activation. In addition, a marked reduction of calcium deposition and BMP-2 expression related to calcium metabolism was found in the statin-treated groups. We found the same strong effects on hypercholesterolemia, antiinflammatory reactions, and BMP-2 expression in the current study.

It is well known that BMP is the most important factor in calcification signaling pathways, and it has been shown that BMP signaling is required for the development of aortic 


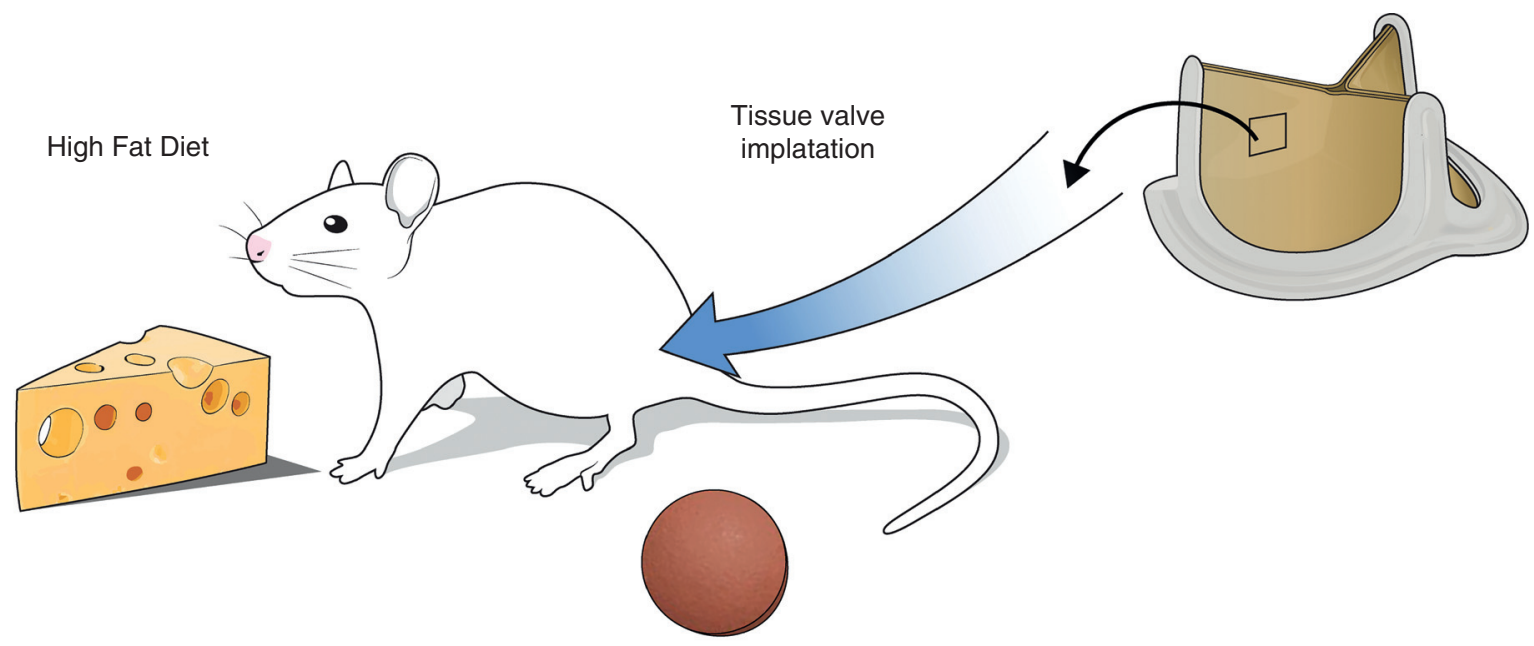

Rosuvastatin

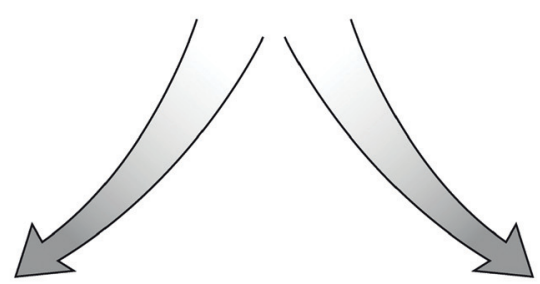

cellular infiltration

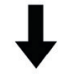

BMP-2 and IL-6 expressions
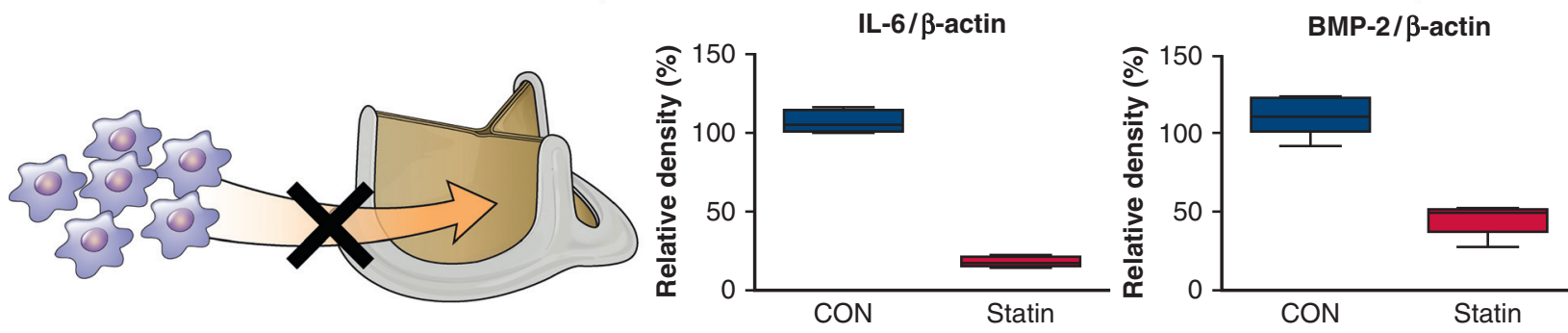

FIGURE 7. Statin attenuated bioprosthetic valve calcification. $B M P-2$, Bone morphogenetic protein 2 ; $I L$, interleukin.

valve calcification in vitro and in vivo. ${ }^{20}$ Statins can strongly inhibit inflammatory mediators such as IL-6, and this anti-inflammatory mechanism might consequentially downregulate BMP-2 in valve tissue. Osman and colleagues $^{21}$ suggested that interstitial cells in human valves are capable of osteoblastic differentiation by potential mediators that could be pharmacologically targeted by statins to cause the downregulation of BMP-2. This suggestion is in accord with our findings of BMP-2 downregulation. Finally, aortic valve stenosis is also characterized by accumulation of oxidized LDL and T-lymphocytes, thus filling tissue inflammation and the release of proinflammatory cytokines such as tumor necrosis factor- $\alpha$ and IL- $1 \alpha$, which in turn induce cell proliferation. Calcification and osteogenesis, mediated by inflammation and processes involving metalloproteinases, have been identified as players in the pathogenesis of aortic stenosis, so statin as a strong antiinflammation agent might play an important role for slowing or preventing calcification. ${ }^{22}$

\section{Study Limitations}

The limitations of this study are the relatively small number of animals used in the experiments and the fact that tissue implantation in a subcutaneous animal model has some differences from the blood circulation model. Therefore, our data should be interpreted with caution. Juvenile sheep are widely used because tests can be done in the systemic circulation. However, these are complex, time-consuming, and expensive. Easier and less-expensive study setups as a first step in developing tissue-engineered heart valves are 
needed. Second, the subdermal model provides excellent contact of host tissue with implanted specimens leading to rapid cellular infiltration. Because of the subdermal position, an absence of bloodstream and mechanical stress occurs, which influences cellular repopulation. Because of these limitations, the rat subdermal model can only provide basic information about immunologic reactions and recellularization. ${ }^{23}$ In addition, the resected prosthetic valve cusps were already injured at the time of cutting, so the continuity of the coated membrane was broken before implantation. Compared with unresected valve cusps, this experimental situation might favor cellular infiltration from the host system, which can induce inflammation and calcification. Third, in this experiment, we did not set and measure the calcification degree in the normal cholesterol group with or without statin for each bioprosthetic, so we cannot decide which is the more dominant factor for reducing calcification between the anti-lipid and anti-inflammatory effects. Finally, the relationship between tissue valve calcification mitigation in animal model studies and clinical outcomes has not been established.

\section{CONCLUSIONS}

High cholesterol levels may be a significant risk factor for the calcification of any type of bioprosthetic aortic valve. Statin treatment significantly attenuated the calcification of bovine and porcine bioprosthetic aortic valves in a subcutaneous rat model and had dual anticalcification and anti-inflammation effects. Our results suggest that statin treatment might be helpful for the longevity of BHVs.

\section{Conflict of Interest Statement}

Authors have nothing to disclose with regard to commercial support.

The authors thank the Yonsei Medical Research Center for animal care and breeding the rat model, and Dong-Su Jang, MFA (Medical Illustrator), for help with the illustrations.

\section{References}

1. Cohn LH, Collins JJ Jr, Rizzo RJ, Adams DH, Couper GS, Aranki SF. Twentyyear follow-up of the Hancock modified orifice porcine aortic valve. Ann Thorac Surg. 1998;66(6 Suppl):S30-4.

2. Jamieson WR, Rosado LJ, Munro AI, Gerein AN, Burr LH, Miyagishima RT, et al. Carpentier-Edwards standard porcine bioprosthesis: primary tissue failure (structural valve deterioration) by age groups. Ann Thorac Surg. 1988;46:155-62.

3. Schoen FJ. Cardiac valve prostheses: review of clinical status and contemporary biomaterials issues. J Biomed Mater Res. 1987;21(A1 Suppl):91-117.
4. Colli A, Gherli T, Mestres CA, Pomar JL. Degeneration of native and tissue prosthetic valve in aortic position: do statins play an effective role in prevention? Int $J$ Cardiol. 2007;116:144-52.

5. Farivar RS, Cohn LH. Hypercholesterolemia is a risk factor for bioprosthetic valve calcification and explantation. J Thorac Cardiovasc Surg. 2003;126: 969-75.

6. Aronow WS, Ahn C, Kronzon I, Goldman ME. Association of coronary risk factors and use of statins with progression of mild valvular aortic stenosis in older persons. Am J Cardiol. 2001;88:693-5.

7. Achenbach S, Ropers D, Pohle K, Leber A, Thilo C, Knez A, et al. Influence of lipid-lowering therapy on the progression of coronary artery calcification: a prospective evaluation. Circulation. 2002;106:1077-82.

8. Palta S, Pai AM, Gill KS, Pai RG. New insights into the progression of aortic stenosis: implications for secondary prevention. Circulation. 2000;101:2497-502.

9. Black AE, Sinz MW, Hayes RN, Woolf TF. Metabolism and excretion studies in mouse after single and multiple oral doses of the 3-hydroxy-3-methylglutarylCoA reductase inhibitor atorvastatin. Drug Metab Dispos. 1998;26:755-63.

10. Lee SH, Kim DH, Youn YN, Lee S, Joo HC, Chang BC, et al. Effect of rosuvastatin on bovine pericardial aortic tissue valve calcification in a rat subdermal implantation model. Korean Circ J. 2017;47:401-8.

11. Neto-Ferreira R, Novaes Rocha V, da Silva Torres T, Mandarim-de-Lacerda CA, de Carvalho JJ. Beneficial effects of rosuvastatin on aortic adverse remodeling in nitric oxide-deficient rats. Exp Toxicol Pathol. 2011;63:473-8.

12. Gilmanov D, Bevilacqua S, Mazzone A, Glauber M. Do statins slow the process of calcification of aortic tissue valves? Interact Cardiovasc Thorac Surg. 2010; 11:297-301.

13. Bellamy MF, Pellikka PA, Klarich KW, Tajik AJ, Enriquez-Sarano M. Association of cholesterol levels, hydroxymethylglutaryl coenzyme-A reductase inhibitor treatment, and progression of aortic stenosis in the community. J Am Coll Cardiol. 2002;40:1723-30.

14. Kulik A, Masters RG, Bedard P, Hendry PJ, Lam BK, Rubens FD, et al. Postoperative lipid-lowering therapy and bioprosthesis structural valve deterioration: justification for a randomised trial? Eur J Cardiothorac Surg. 2010;37:139-44.

15. Lorusso R, Corradi D, Maestri R, Bosio S, Curulli A, Beghi C, et al. Atorvastatin attenuates post-implant tissue degeneration of cardiac prosthetic valve bovine pericardial tissue in a subcutaneous animal model. Int J Cardiol. 2010;141:68-74.

16. Price L, Sniderman A, Omerglu A, Lachapelle K. Bioprosthetic valve degeneration due to cholesterol deposition in a patient with normal lipid profile. Can J Cardiol. 2007;23:233-4.

17. Demer LL. Cholesterol in vascular and valvular calcification. Circulation. 2001 104:1881-3.

18. Olsson M, Thyberg J, Nilsson J. Presence of oxidized low density lipoprotein in nonrheumatic stenotic aortic valves. Arterioscler Thromb Vasc Biol. 1999;19: 1218-22.

19. Schmermund A, Mohlenkamp S, Erbel R. The latest on the calcium story. Am J Cardiol. 2002;90:12L-4L.

20. Lin CP, Huang PH, Lai CF, Chen JW, Lin SJ, Chen JS. Simvastatin attenuates oxidative stress, NF-kappaB activation, and artery calcification in LDLR-/mice fed with high fat diet via down-regulation of tumor necrosis factor-alpha and TNF receptor 1. PLoS One. 2015;10:e143686.

21. Osman L, Yacoub MH, Latif N, Amrani M, Chester AH. Role of human valve interstitial cells in valve calcification and their response to atorvastatin. Circulation. 2006;114(1 Suppl):I547-52.

22. Akin I, Nienaber CA. Is there evidence for statins in the treatment of aortic valve stenosis? World J Cardiol. 2017:9:667-72.

23. Christ T, Dohmen PM, Holinski S, Schonau M, Heinze G, Konertz W. Suitability of the rat subdermal model for tissue engineering of heart valves. Med Sci Monit Basic Res. 2014;20:194-9.

Key Words: heart valve, hypercholesterolemia, statins (HMG-CoA reductase inhibitor), aortic valve, calcification 


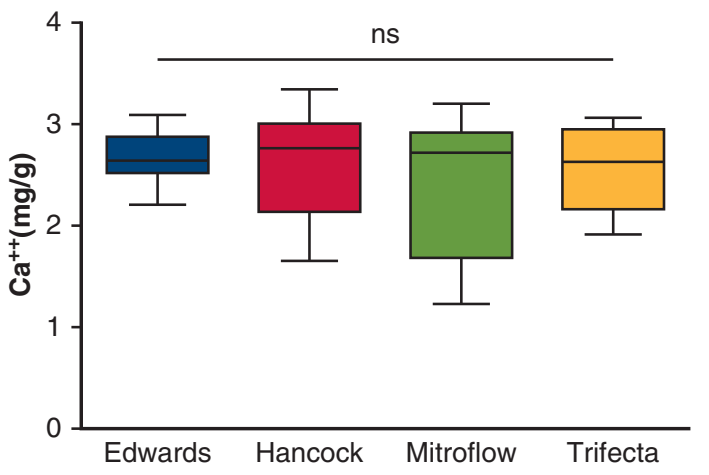

FIGURE E1. Various commercially available BHV calcification results. $n s$, Not significant. 